LA GRANJA:

REVISTA DE

CIENCIAS DE LA VIDA

pISSN:1390-3799; eISSN:1390-8596

http:/ / doi.org/10.17163/lgr.n33.2021.01
Artículo científico / Scientific paper

LA COMPRENSIÓN DE LA VACA LECHERA

PARA UNA PRODUCCIÓN SUSTENTABLE

\title{
PROTOCOLO J-SYNCH CON Y SIN ECG EN VACAS BROWN SWISS Y SUS CRUZAS CON BOS INDICUS EN LA AMAZONÍA ECUATORIANA
}

\author{
PROTOCOLS J-SYNCH WITH AND WITHOUT ECG IN BROWN SWISS AND \\ CROSSES WHITH BOS INDICUS COWS IN THE ECUADORIAN AMAZONS

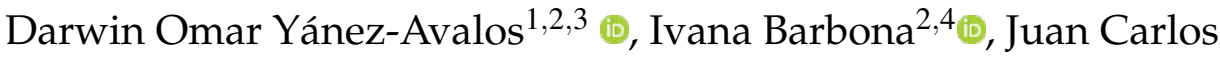 \\ López-Parra ${ }^{2,5}$ y Pablo Roberto Marini ${ }^{* 2,6,7}$
}

${ }^{1}$ Maestría en Reproducción Bovina. Instituto de Reproducción Animal y Universidad Nacional de Córdoba, Av. Haya de la Torre s/n, Córdoba, Argentina.

2 Facultad de Ciencias Veterinarias. Centro Latinoamericano de Estudios de Problemáticas Lecheras, Universidad Nacional de Rosario, Ruta 33 y Ovidio Lagos, 2170 Casilda, Santa Fe, Argentina.

${ }^{3}$ Ministerio de Agricultura y Ganadería, Av. Amazonas, Quito, 170517, Ecuador.

${ }^{4}$ Facultad de Ciencias Agrarias. Universidad Nacional de Rosario, Ruta 33 y Ovidio Lagos, 2170 Casilda, Santa Fe, Argentina.

${ }^{5}$ Centro Nacional de Mejoramiento Genético y Productivo "El Rosario", Subsecretaría de desarrollo Pecuario, Ministerio de Agricultura y Ganadería, Ecuador.

${ }^{6}$ Facultad de Ciencias Veterinarias. Universidad Nacional de Rosario, Ruta 33 y Ovidio Lagos, 2170 Casilda, Santa Fe, Argentina.

${ }^{7}$ Carrera de Investigador Científico (CIC). Universidad Nacional de Rosario, CGK, Maipú 1065, S2000, Rosario, Santa Fe, Argentina.

*Autor para correspondencia: pmarini@unr.edu.ar

Manuscrito recibido el 04 de abril de 2020. Aceptado, tras revisión, el 13 de septiembre de 2020. Publicado el 1 de marzo de 2021.

\section{Resumen}

La incorporación de protocolos con proestro prolongado en condiciones extremas ha permitido mejorar la concepción. Por lo tanto, el objetivo de esta investigación fue evaluar los resultados del protocolo con proestro prolongado denominado J-Synch con y sin eCG, sobre la dinámica folicular y porcentaje de preñez en vacas doble propósito en la Amazonía Ecuatoriana. El trabajo se efectuó en la provincia de Pastaza - Ecuador, desde octubre del 2018 a octubre del 2019. Se utilizaron 448 vacas multíparas en producción con cría al pie de las razas Brown Swiss y sus cruzas (Bos indicus). Se trabajó con cuatro tratamientos, las vacas fueron inseminadas artificialmente a tiempo fijo en el día nueve T1: J-Synch + eCG +Celo IATF 60 horas $(\mathrm{n}=120)$; T2: J-Synch+eCG +Sin Celo+ GnRH IATF 72 horas $(\mathrm{n}=118)$; T3: J-Synch+ Celo IATF 60 horas $(\mathrm{n}=103)$; T4: J-Synch+ Sin Celo+ GnRH +IATF 72 Horas $(\mathrm{n}=107)$. La tasa de preñez fue del $55 \%$ (T1), el $49 \%$ (T2), el $51 \%$ (T3) y el $50 \%$ (T4) sin mostrar diferencias significativas. El desarrollo folicular y 
el diámetro del cuerpo lúteo mostraron diferencias significativas $(\mathrm{p} \leq 0,05)$. Se concluye que los tratamientos J-Synch más eCG a las 60 horas y las 72 horas tuvieron diferente comportamiento en el desarrollo folicular y en el tamaño del cuerpo lúteo. El porcentaje de preñez no difirió entre los tratamientos, aunque existió un 44,5\% más probabilidad de quedar preñada una vaca con el T1 en comparación con el T2. Además, que aumentaría un 22,5\% la preñez aplicando el T1 en comparación con el T3, y se lograría un 18\% mayor de preñez con el T3 si se compara con el T2.

Palabras clave: Dinámica follicular, preñez, progesterona, inseminación artificial a tiempo fijo.

\begin{abstract}
The incorporation of protocols with prolonged proestrus in extreme conditions has allowed to improve pregnancy. The objective was to evaluate the results of the protocol with prolonged proestrus called J-Synch with and without eCG, on follicular dynamics and pregnancy percentage in dual-purpose cows in the Ecuadorian Amazon. The project was developed of the Province of Pastaza - Ecuador, from October 2018 to October 2019. The study used 448 multiparous dual purpose Brown Swiss cows with calves and their crosses (Bos indicus). Four treatments were used, the cows were artificially inseminated at a fixed time on day nine T1: J-Synch+ eCG+ estrus FTAI 60 hours (n=120); T2: J-Synch+ eCG+ without estrus+ GnRH FTAI 72 hours $(\mathrm{n}=118)$; T3: J-Synch+ estrus FTAI 60 hours (n=103); T4: J-Synch+ without estrus + GnRH + FTAI 72 hours $(n=107)$. The pregnancy rate was 55\% (T1), 49\% (T2), 51\% (T3) and $50 \%$ (T4) without showing significant differences. Follicular development and corpus luteum diameter showed significant differences $(\mathrm{p} \leq 0.05)$. It is concluded that the J-Synch treatments plus eCG at 60 hours and 72 hours had different behavior in follicular development and in the size of the corpus luteum. The pregnancy percentage did not differ between treatments. Although, there was a $44.5 \%$ more probability of getting a cow pregnant with T1 compared to T2. Furthermore, pregnancy would increase by $22.5 \%$ applying T1 compared to T3, and pregnancy with T3 would be $18 \%$ higher if compared to T2.
\end{abstract}

Keywords: Follicular dynamics, pregnancy, progesterone, fixed time artificial insemination.

\footnotetext{
Forma sugerida de citar: Yánez-Avalos, D.O., Barbona, I., López-Parra, J.C. y Marini, P.R. (2021). Protocolo J-Synch con y sin eCG en vacas Brown Swiss y sus cruzas con Bos Indicus en la amazonía ecuatoriana. La Granja: Revista de Ciencias de la Vida. Vol. 33(1):8-20. http://doi.org/10.17163/lgr.n33.2021.01.
}

\title{
IDs Orcid:
}

Darwin Omar Yánez-Avalos: http:/ / orcid.org/0000-0001-9306-3489

Ivana Barbona: http:/ /orcid.org/0000-0003-2618-9983

Juan Carlos López-Parra: http:/ /orcid.org/0000-0002-0015-0813

Pablo Roberto Marini: http:/ / orcid.org/0000-0003-0826-0387 


\section{Introducción}

Los sistemas de producción con vacas Brown Swiss y sus cruzas con Bos indicus son comunes en la región tropical húmeda, con una producción leche promedio diaria por vaca de 3 a 9 litros, la duración de la lactación de 120 a 180 días y un intervalo parto-parto de 18 a 24 meses (Rodriguez, Saavedra y Gómez, 2015).

Estos parámetros reproductivos son generados por múltiples factores, entre ellos ambientales (temperaturas elevadas y humedad), fisiológicos y de manejo zootécnico. En la región Amazónica los productores tienen la necesidad de mejorar los resultados reproductivos en las vacas para carne y leche (Moyano y col., 2015).

El protocolo denominado J-Synch ha sufrido un sin número de variaciones, sin alterar su forma inicial como: una dosis intramuscular de Benzoato de Estradiol y la colocación de progesterona a través de un dispositivo intravaginal para lograr sincronizar el comienzo de una nueva onda folicular con menor tiempo de inserción del dispositivo con progesterona de 6 días y ya no de 7 u 8 días, aplicando una GnRH como iniciador de la ovulación, a las 72 horas después se remueve el dispositivo junto con la IATF, asegurando un proestro más duradero (De la Mata y Bó, 2012).

Yánez y col. (2017), mostraron los resultados de preñez de dos protocolos de IATF, inseminando a vacas doble propósito de la Amazonía Ecuatoriana, a las 60 y 72 horas de que se removió el dispositivo intravaginal impregnado con progesterona, donde el protocolo J-Synch más eCG 60 horas tuvo el mejor índice de preñez comparado con el protocolo J-Synch más eCG 72 horas. Cedeño y col. (2019) compararon la dinámica folicular y luteal de un tratamiento alternativo de prolongación de proestro J-Synch de 7 días de progesterona en vaquillonas Bos indicus de carne que recibieron inseminación artificial a tiempo fijo, en la Provincia de Manabí-San Vicente, concluyendo que la extensión de un día más de progesterona en el tratamiento J-Synch de 7 días no altera las características del folículo dominante ovulatorio y el momento de ovulación con relación al protocolo J-Synch de 6 días, aunque en ambos protocolos de proestro prolongado, el intervalo a la ovulación es mayor que en los animales tratados con el protocolo convencional con Cipionato de Estradiol como inductor de la ovulación. Castellanos, Rentería y Maraña (2019) experimentaron en Etzatlán, Jalisco, México, con 211 vacas (90 vacas Brahman y 121 vacas F1 Brahman $\times$ Pardo Suizo) sin cría al pie con más de 180 días posparto, con un estado corporal de 3 a 4. El Día 6, 105 vacas fueron asignadas al azar al Grupo J-Synch y las vacas restantes (106), fueron asignadas al Grupo Tradicional, los dos grupos con eCG. No hubo diferencias significativas $(\mathrm{p} \geq 0.05)$ en el porcentaje de preñez entre los grupos. Además, no mostró diferencias significativas $(p \geq 0.05)$ en el porcentaje de preñez de la raza o el toro utilizado.

Con base en los hallazgos de la aplicación de J-Synch, (Bó y col., 2018) propusieron que las vaquillonas que manifiestan celo antes, podrían ser inseminadas antes sin afectar el porcentaje de preñez, pero sería necesario determinar el tiempo óptimo para realizar la IATF en aquellas que no muestran celo. Se realizó un trabajo en donde 1.283 vaquillas cruzadas Angus y Hereford fueron tratadas con el protocolo J-Synch y las vaquillonas se pintaron en la base de la cola cuando se retiró el dispositivo de progesterona. Las vaquillonas recibieron $\mathrm{GnRH}$ / IATF a las 60 o $72 \mathrm{~h}$, independientemente de la eliminación de pintura. La preñez fue mayor en aquellos que mostraron estro antes de la IATF que en aquellos que no lo hicieron, independientemente del tiempo de inseminación $(53.6 \%, 542 / 1012$ vs. $45.0 \%, 122 / 271$, respectivamente, $\mathrm{P} \leq 0.05$ ).

La implicación práctica de este resultado es que cuando se sincronizan grandes rebaños, el dispositivo podría retirarse en la tarde del día 6 y la IATF comienza a las 60 h (día 9 a.m.) en todas las vaquillonas con pintura alterada; y aquellas con la pintura intacta podrían separarse para recibir $\mathrm{GnRH}$ en el momento de la inseminación artificial a tiempo fijo en la tarde del día 9 (es decir, alrededor o después de 72 horas).

La incorporación de protocolos con proestro prolongado ha permitido aumentar los índices de preñez en vacas anéstricas, con días abiertos elevados y con cría al pie en climas tropicales. Sin embargo, el protocolo J-Synch es necesario, aunque se siga validando en condiciones de la Amazonía Ecuatoriana. El objetivo fue evaluar los resultados del protocolo con proestro prolongado denominado J- 
Synch con y sin eCG, en el comportamiento folicular y el porcentaje de preñez, en vacas Brown Swiss y sus cruzas con Bos indicus en la Amazonía Ecuatoriana.

\section{Materiales y Métodos}

El trabajo se realizó en Pastaza, provincia de Ecuador, iniciando en octubre de 2018 y finalizando en octubre de 2019. En la Amazonía las precipitaciones son de 4000-5000 mm/año, elevada humedad y calor con temperaturas que oscilan entre los $18 \mathrm{y}$ $24{ }^{\circ} \mathrm{C}$. Los productores poseen fincas de 50 ha promedio en terrenos irregulares, utilizando como forrajes el Axonopusscoparius (Gramalote) (GonzálezMarcillo y col., 1997).

Se contó con 448 vacas de las razas Brown Swiss y sus cruzas Bos indicus, alimentadas mediante un sistema de pastoreo al sogueo (Carrera Durazno, Fierro y Ordoñez, 2015), rango de 90 a 132 días de posparto, multíparas, edad de entre 34 y 65 meses, condición corporal de 2,5 a 3 (escala del 1 al 5, Ben y col. (2002)), un peso de entre 350 y $380 \mathrm{~kg}$, y fueron seleccionadas por la presencia de un folículo $>8$ $\mathrm{mm}$ de diámetro en sus ovarios por medio de ultrasonografía. Se utilizaron pajillas de semen de 0,50 $\mathrm{ml}$ de raza Brown Swiss previamente analizado en las IATF.

\subsection{Tratamientos}

Se utilizó el protocolo de sincronización de celos con proestro prolongado denominado J-Synch (De la Mata y Bó, 2012) el cual se modifica adicionando eCG y realizando la IATF a las 60 y 72 horas de retirado el dispositivo intravaginal. El total de vacas se dividió aleatoriamente en dos grupos: Grupo 1 con adición de eCG y Grupo 2 sin eCG. A su vez estos dos grupos se subdividieron en dos grupos más, uno que se realiza IATF a las 60 horas y otro la IATF a las 72 horas de haber retirado el dispositivo intravaginal. Esquemáticamente representado se lo representa así:

$$
\text { T1: J-Synch + eCG + Celo - IA } 60 \text { horas }(\mathrm{n}=120)
$$

T2: J-Synch + eCG + Sin Celo + GnRH - IATF 72 horas $(n=118)$

\author{
T3: J-Synch + Celo - IA 60 horas $(\mathrm{n}=103)$ \\ T4: J-Synch+ Sin Celo + GnRH - IATF 72 Horas \\ $(\mathrm{n}=107)$
}

La distribución de los tratamientos (T) fue completamente al azar, en cada uno se realizaron cuatro repeticiones (T1: 30, 30, 30 y 30 (120), T2: 29, 29, 30 y 30 (118), T3: 25, 26, 26 y 26 (103) T4: 26, 27, 27 y 27 (107) vacas con cada uno de los tratamientos).

Para el desarrollo de la investigación, en el día 0 a cada una las 306 vacas se realizó una primera evaluación reproductiva mediante ultrasonografía para analizar el estatus ovárico y que todos los animales cumplan con los parámetros propuestos para poder ser incluidas en la presente investigación; adicionalmente, se le colocó un dispositivo intravaginal con progesterona (DIB $0.5 \mathrm{gr}$ ) más la aplicación de $2 \mathrm{mg}$ de BE por vía IM; al día 6 las vacas se dividen en grupos según los tratamientos así:

\subsubsection{Tratamiento 1}

Referencias: En el día 0 se realizó la primera evaluación ecográfica para analizar el estatus ovárico y se aplicó BE (2 mg de Benzoato de Estradiol) por vía IM junto con un dispositivo intravaginal DIB 0,5g. El día 6 se retiró el dispositivo con la administración de $\mathrm{PGF}_{2 \alpha}$ (500 $\mu \mathrm{g}$ de Cloprostenol) más la administración de eCG (400UI de Gonadotropina coriónica equina) y se dividió en dos grupos: $T 1$ (120 vacas) y T2 (118 vacas), se realizó una medición con ultrasonografía del desarrollo folicular al inicio del proestro, y se aplicó un marcador de pintura en la base de la cola para observar la presencia de celo previo a la IATF (Inseminación artificial a tiempo fijo) (Figura 1).

Los animales que presentaron celo antes de las 60 horas fueron inseminados a las 60 horas (T1) y los que no, IATF a las 72 horas más la adición de GnRH (100 $\mu$ g acetato de gonadorelina) (T2). Al día 9 o final del proestro se continuó con la medición ultrasonográfica para ver el comportamiento de los folículos. Seguido de esto, a partir de las 60 horas de retirado el dispositivo intravaginal, mediante ultrasonografía cada 12 horas, se determinó el momento de la ovulación en los dos tratamientos. Al día 
7 post inseminación se midió por medio de ultrasonografía el tamaño de cuerpo lúteo y la calidad a través de una muestra sérica y se analizaron los niveles de progesterona ( $\mathrm{ng} / \mathrm{ml})$. El diagnóstico de gestación se realizó a los 35 a 40 días posteriores a las IATF mediante ultrasonografía (ecógrafo veterinario Ibex - pro con sonda lineal $5 \mathrm{MH}$ en modo V).

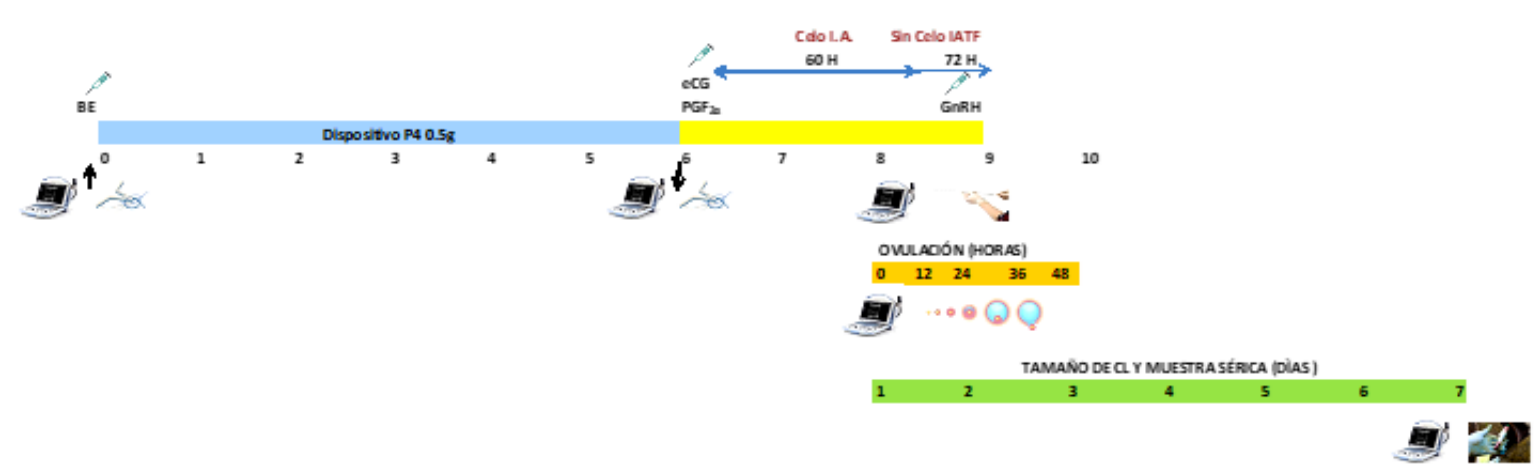

Figura 1. T1: J-Synch + eCG + Celo - I.A. 60 horas. Sin Celo I.A.T.F. 72 Horas + GnRH.

\subsubsection{Tratamiento 2}

Referencias: En el día 0 se realizó la primera evaluación ecográfica para analizar el estatus ovárico y se aplicó BE (2 mg de Benzoato de Estradiol) por vía IM junto con un dispositivo intravaginal DIB 0,5g. El día 6 se retiró el dispositivo con la administración de $\mathrm{PGF}_{2 \alpha}$ (500 $\mu \mathrm{g}$ de Cloprostenol) y se dividió en dos grupos: T3 (103 vacas) y T4 (107 vacas), se realizó una medición con ultrasonografía del desarrollo folicular al inicio del proestro, y se aplicó un marcador de pintura en la base de la cola para observar la presencia de celo previo a la IATF (Inseminación artificial a tiempo fijo). Los animales que presentaron celo antes de las 60 horas fueron inseminados a las 60 horas (T3) y los que no, IATF a las
72 horas más la adición de GnRH (100 $\mu$ g acetato de gonadorelina) (T4). Al día 9 o final del proestro se continuó con la medición ultrasonografía para ver el comportamiento de los folículos. Seguido de esto, a partir de las 60 horas de retirado el dispositivo intravaginal, mediante ultrasonografía cada 12 horas, se determinó el momento de la ovulación en los dos tratamientos. Al día 7 post inseminación se midió por medio de ultrasonografía el tamaño de cuerpo lúteo y la calidad a través de una muestra sérica y se analizaron los niveles de progesterona (ng/ml). El diagnóstico de gestación se realizó a los 35 a 40 días posteriores a las IATF mediante ultrasonografía (ecógrafo veterinario Ibex-Pro con sonda lineal 5 $\mathrm{MH}$ en modo V) (Figura 2).

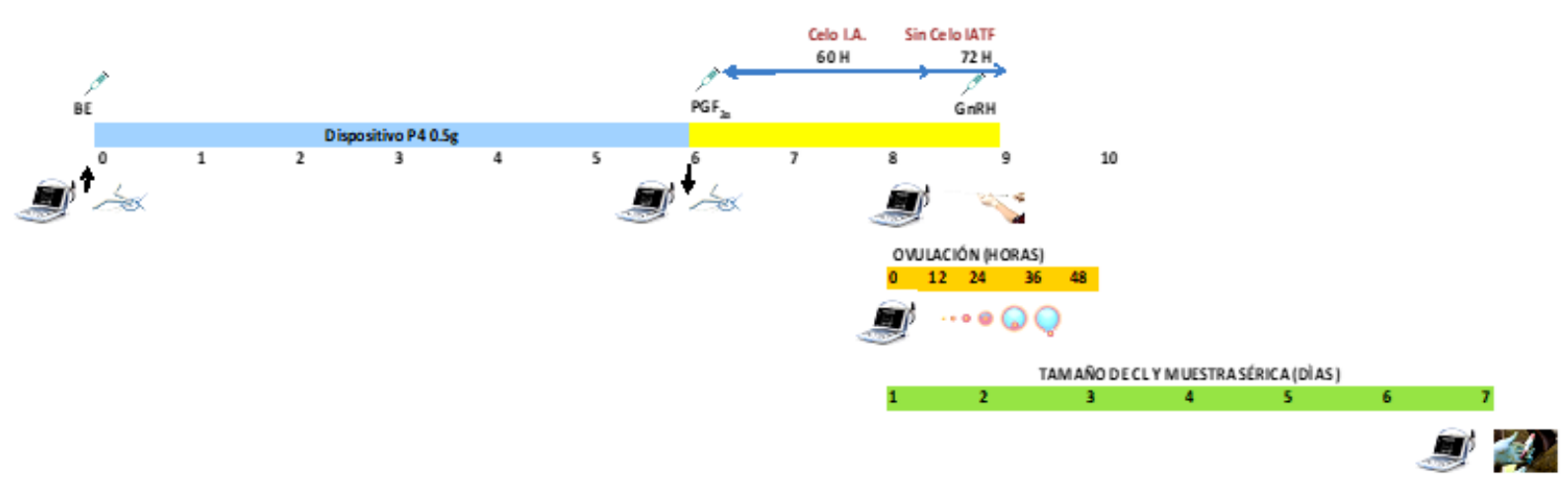

Figura 2. T2: J-Synch + Celo - I.A. 60 horas. Sin Celo I.A.T.F. 72 Horas $+\mathrm{GnRH}$ 
Para la IATF se utilizó semen congelado/descongelado utilizando la técnica descrita por Bernardi y col. (2011), de un solo toro y de fertilidad probada, aunque igualmente antes de utilizarlo se le realizó un análisis en el Laboratorio de Biotecnología del Centro de Investigación, Posgrado y Conservación Amazónica y fueron inseminadas por un mismo técnico. Se observaron los celos a través de dos comportamientos en la revisación: despintadas las bases de la cola y el moco vaginal (clara de huevo). Esta observación se realizó antes de iniciar la ecografía e IATF. Además, la detección del celo comenzó 24 horas después de retirado el dispositivo, dos veces en el día (mañana y tarde) los días 7,8 y 9.

\subsection{Dinámica folicular y luteal}

El desarrollo folicular fue evaluado a través de ultrasonografía en el día seis cuando se retiró el dispositivo intravaginal de progesterona, también en el momento de realizar la IATF (60 y 72 horas de retirado el dispositivo intravaginal de progesterona), con el objetivo de realizar un seguimiento del comportamiento ovárico y al final del celo. Además, al día siete posterior a la inseminación a través de ecografía se tomaron las medidas del tamaño del cuerpo lúteo, utilizando lo recomendado por Kastelic, Bergfelt y Ginther (1990), sobre dos medidas (alto-ancho en $\mathrm{mm}$ ) de cada estructura (cuerpo lúteo o folículo) para luego obtener el promedio entre ambas.

Para determinar el momento de la ovulación (desaparición del folículo de mayor diámetro) se continuó evaluando mediante ultrasonografía el desarrollo folicular, esto se realizó en un grupo menor de vacas $\mathrm{T} 1$ y T2 $=24$; $\mathrm{T} 3$ y T4 $=18$ debido a las dificultades para acceder o permanecer en diferentes horarios en los establecimientos utilizados. Para ello se realizó una ecografía 12 horas posterior de la IATF, luego a los animales que aún no habían registrado celo se realizó otra ultrasonografía a las 24 horas y a los animales que aún no habían ovulado hasta esta instancia se determinó subjetivamente que ovularon $>24$ horas posteriores a la IATF lo que se reconfirmó con la presencia del CL a los 7 días pos IATF.

La gestación se determinó a través de ultrasonografía entre los días 40 a 45 días de realizada la
IATF (Ibex Pro 2011 and Lyte, USA, transductor L6.2 with a linear probe of $5.0 \mathrm{MHz}$ )

\subsection{Variables analizadas}

- Detección de celos (presencia o ausencia).

- Desarrollo folicular (en mm).

- Momento de la ovulación (en horas).

- Tamaño de cuerpo lúteo post-ovulación (en $\mathrm{mm})$.

- Preñez: presencia o ausencia

- Tiempo: día 6 y día 8.

\subsection{Análisis estadístico}

Para el ajuste se utilizó un modelo de Regresión Logística Múltiple para estudiar la preñez (presencia o ausencia) en función de los diferentes protocolos, días, desarrollo folicular en $\mathrm{mm}$, y tamaño del cuerpo lúteo post-ovulación en $\mathrm{mm}$. Este modelo permite tener en cuenta la naturaleza binaria de la variable respuesta. La interpretación del modelo se realizó mediante el análisis de las Razones de Odds estimada, junto con sus intervalos de confianza. Para los análisis estadísticos se utilizó el programa JMP versión 5.0 para Windows.

\section{Resultados}

\subsection{Porcentaje de preñez}

De las 448 vacas inseminadas, se preñaron 230 $(51.3 \%)$ vacas y quedaron vacías $218(48.6 \%)$ vacas. El porcentaje de preñez de los protocolos T1, T2, T3 y T4 no mostró diferencias significativas $(\mathrm{P} \geq 0.7989)$ (Tabla 1).

\subsection{Expresión de celo}

Existió un 51\% (230/448) de presencia de celo del total de animales para ambos tratamientos (Tabla 2). El porcentaje de animales con presencia de celo en el tratamiento J-Synch con eCG (T1 y T2) fue 52.1\% $(124 / 238)$ y en el tratamiento J-Synch sin eCG (T3 y T4) fue 50\% (106/210), sin diferencias significativas $(\mathrm{P} \geq 0.05)$. El porcentaje de preñez en el protocolo JSynch con eCG + I.A. 60 horas en las vacas que presentaron celo fue del $55 \%(66 / 120)$ T1 y J-Synch sin 
eCG + I.A. a las 60 horas (T3) fue del $54 \%(39 / 72)$, los cuales no mostraron diferencia significativa, al igual que las vacas sin presencia de celo $+\mathrm{GnRH}+$ IATF 72 horas que fueron $49 \%$ (58/118) T2 y $49 \%$ $(67 / 138) T 4$, respectivamente $(\mathrm{P} \geq 0.05)$.

\subsection{Dinámica folicular en diferentes mo- mentos}

El tamaño folicular cuando se retiró del dispositivo evidenció diferencias significativas $(\mathrm{p} \leq 0.05)$ con mayor diámetro para T2 $(9.7 \pm 0.1 \mathrm{~mm})$. De la misma manera, el tamaño del folículo al inicio de la inseminación artificial manifestó diferencias significativas $(\mathrm{P} \leq 0.05)$ con un mayor diámetro para $T 4$ $(13.2 \pm 0.1 \mathrm{~mm})$ y cuando finalizó el estro, el tamaño del folículo mostró diferencias significativas $(\mathrm{P} \leq 0.05)$ con un mayor diámetro para T3 $(13.9 \pm 0.2$ $\mathrm{mm})$.

Tabla 1. Porcentaje de preñez en vacas Brown Swiss y sus cruzas sometidas a tratamientos J-Synch con y sin eCG, a las 60 y 72 horas de IATF.

\begin{tabular}{cccc}
\hline Tratamientos & $\mathbf{N}^{\circ}$ animales & $\begin{array}{c}\text { Animales } \\
\text { preñados }\end{array}$ & $\%$ preñez \\
\hline T1 & 120 & $(66 / 120)$ & 55 \\
\hline T2 & 118 & $(58 / 118)$ & 49 \\
\hline T3 & 103 & $(53 / 103)$ & 51 \\
\hline T4 & 107 & $(53 / 107)$ & 50 \\
\hline Total & 448 & $(230 / 448)$ & 51.3 \\
\hline
\end{tabular}

Tabla 2. Manifestación del celo en vacas Brown Swiss y sus cruzas sometidas al tratamiento J-Synch con eCG y sin eCG.

\begin{tabular}{cccc}
\hline Tratamientos & $\mathbf{N}^{\circ}$ animales & Con celo & Sin celo \\
\hline J-Synch con eCG & 238 & $124(52.1 \%)$ & $114(47.8 \%)$ \\
\hline J-Synch sin eCG & 210 & $106(50.5 \%)$ & $104(49.5 \%)$ \\
\hline TOTAL & 448 & $230(51.3 \%)$ & $218(48.6 \%)$ \\
\hline
\end{tabular}

Tabla 3. Dinámica folicular (mm) en los cuatro protocolos utilizados en vacas Brown Swiss y sus cruzas. Promedios y errores estándar

\begin{tabular}{cccc}
\hline \multicolumn{4}{c}{ Tamaño del folículo en mm } \\
\hline $\begin{array}{c}\text { Tratamiento / } \\
\text { Momento }\end{array}$ & $\begin{array}{c}\text { Al retiro del retiro } \\
\text { del dispositivo }\end{array}$ & $\begin{array}{c}\text { A la inseminación } \\
\text { artificial }\end{array}$ & $\begin{array}{c}\text { Al finalizar } \\
\text { el estro }\end{array}$ \\
\hline T1 & $9.3 \pm 0.1 \mathrm{ab}$ & $12.8 \pm 0.1 \mathrm{a}$ & $13.0 \pm 0.2 \mathrm{~b}$ \\
$\mathrm{~T} 2$ & $9.7 \pm 0.1 \mathrm{a}$ & $12.1 \pm 0.1 \mathrm{~b}$ & $12.8 \pm 0.2 \mathrm{~b}$ \\
$\mathrm{~T} 3$ & $9.3 \pm 0.1 \mathrm{ab}$ & $12.2 \pm 0.1 \mathrm{~b}$ & $13.9 \pm 0.2 \mathrm{a}$ \\
$\mathrm{T} 4$ & $9.1 \pm 0.1 \mathrm{~b}$ & $13.2 \pm 0.1 \mathrm{a}$ & $12.5 \pm 0.8 \mathrm{ab}$ \\
\hline \multicolumn{4}{l}{ Nota: diferentes letras en cada columna indican diferencias significativas $(\mathrm{P} \leq 0.05)}$.
\end{tabular}

\subsection{Momento de la ovulación}

La ovulación, desde la remoción del dispositivo, fue mayor $(\mathrm{F}=18.2 ; \mathrm{P} \leq 0,001)$ para el tratamiento J-Synch con eCG (T1 y T2) $(94.5 \pm 0.9)$ horas, que para el grupo J-Synch sin eCG $(88.0 \pm 1.1)$ horas.
En la Figura 3 se observa la respuesta de la ovulación y tasa de preñez registrados en porcentaje, a las 84, 96 y 108 horas de realizada la IATF en vacas Brown Swiss y sus cruzas con el tratamiento J-Synch 
sin eCG. Mientras que en la Figura 4 se observa la respuesta de la ovulación y tasa de preñez registrados en porcentaje, a las 84,96 y 108 horas de realiza- da la IATF en vacas Brown Swiss y sus cruzas bajo el tratamiento J-Synch con eCG.

Tabla 4. Respuesta de la ovulación posterior a las 24 horas de la IATF en vacas Brown Swiss y sus cruzas según los tratamientos de J-Synch con y sin eCG.

\begin{tabular}{cccc}
\hline Tratamientos & $\mathbf{N}^{\circ}$ animales & $\begin{array}{c}\text { Horas de } \\
\text { ovulación }\end{array}$ & \% de ovulación \\
\hline J- Synch con eCG & 24 & $94.5 \pm 0.9$ & 51.0 \\
J-Synch sin eCG & 18 & $88.0 \pm 1.1$ & 48.5 \\
\hline TOTAL & 42 & $91.25 \pm 1.0$ & 50.0 \\
\hline
\end{tabular}

Figura 3. Respuesta de ovulación y tasa de preñez con tratamiento J-Synch con eCG.

口Ovula 口Preñada $\square \%$ Preñez
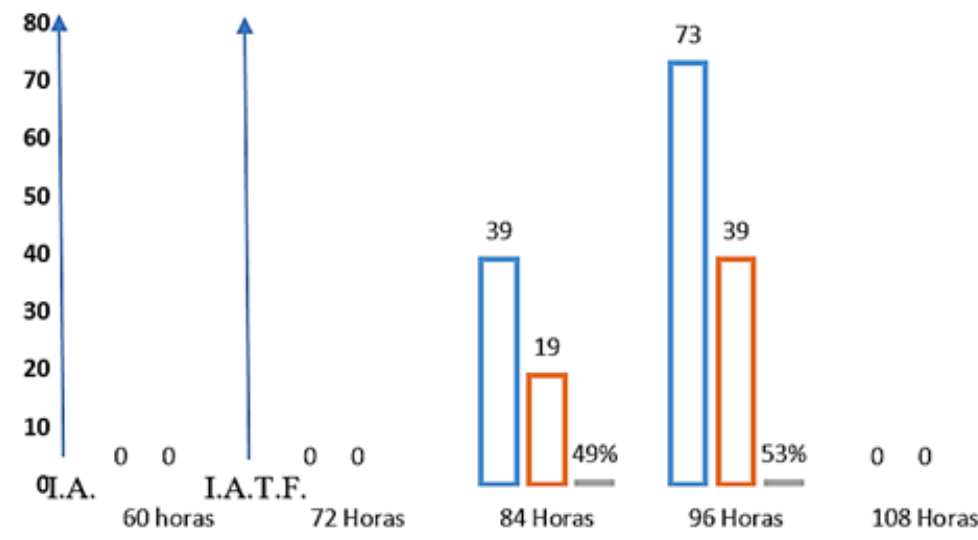

Figura 4. Respuesta de ovulación y tasa de preñez con tratamiento J-Synch sin eCG.

口Ovula 口Preñada $\square \%$ Preñez

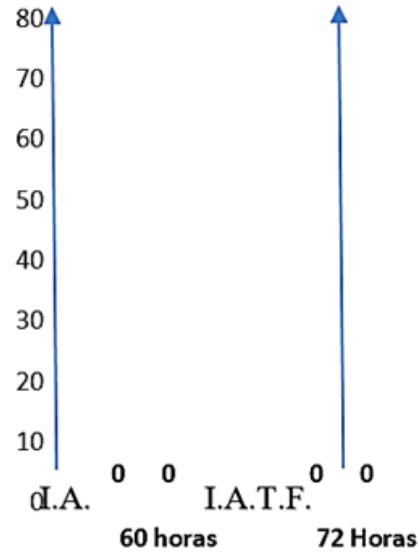

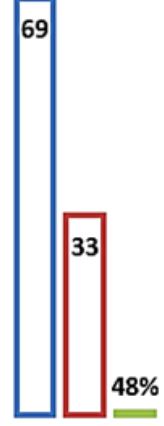

84 Horas

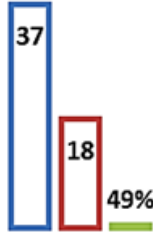

96 Horas
$0 \quad 0$

108 Horas 


\subsection{Tamaño del cuerpo lúteo}

El cuerpo lúteo fue medido (su diámetro en mm) al día 7 post inseminación, difiriendo significativamente entre los tratamientos $(\mathrm{P} \leq 0.05)(\mathrm{T} 1: 21.6 \pm$ $0.2 \mathrm{~b} \mathrm{~mm}$; T2: $22.0 \pm 0.2 \mathrm{~b} \mathrm{~mm}$; T3: $22.1 \pm 0.2 \mathrm{~b} \mathrm{~mm}$ y T4: $25.0 \pm 0.2 \mathrm{a} \mathrm{mm}$ ). En la Tabla 5 se puede observar que las variables colaboran significativamente al modelo, lo que significa que el porcentaje de preñez está significativamente afectado por el protocolo utilizado, el tamaño del folículo dominante y el tamaño del cuerpo lúteo.

Tabla 5. Interacción de las variables frente a la tasa de preñez.

\begin{tabular}{cccc}
\hline Efecto & DF & $\begin{array}{c}\chi^{2} \\
\text { de Wald }\end{array}$ & $\operatorname{Pr}>\chi^{2}$ \\
\hline Protocolo & 3 & 372.609 & $<.0001$ \\
Día & 1 & 298.451 & $<.0001$ \\
TF mm & 1 & 461.369 & $<.0001$ \\
TCL mm & 1 & 739.256 & $<.0001$ \\
\hline
\end{tabular}

Todas las variables resultan estadísticamente significativas en el modelo $(\mathrm{P} \leq 0.001$ en todos los casos). Existe un $44.5 \%$ más probabilidad de quedar preñada una vaca con el T1 (con eCG 60 hs) en comparación con el T2 (con eCG 72 hs). Aumentaría un $22.5 \%$ más la tasa de concepción aplicando el T1 (con eCG 60 hs) en comparación con el T3 (sin eCG 60hs). Sería un $18 \%$ mayor la preñez con el T3 (sin eCG 60hs) si se compara con el T2 (con eCG 72hs). En todos estos casos, los intervalos de confianza de la RO estimadas incluyen el valor 1 . Es decir, que podrían ser casos en los que las chances de preñez en ambos grupos sean iguales, y la diferencia se deba al azar (dado que una $\mathrm{RO}=1$ significa que la chance de quedar preñada es la misma para las dos categorías que se estén comparando).

La probabilidad de quedar preñada es cuatro veces más en el T1 (con eCG 60hs) al compararlo con el T4 (sin eCG 72 hs). Es casi tres veces más la probabilidad de preñez con el T2 (con eCG 72hs) en comparación con el T4 (sin eCG 72hs). Es 3.5 veces más en el T3 (sin eCG 60hs) cuando se compara con la misma chance en el T4 (sin eCG 72hs).

A los ocho días es casi cuatro veces mayor preñar una vaca que la misma chance a los seis días. Existe un $50 \%$ mayor de probabilidad de preñar cuando aumenta $1 \mathrm{~mm}$ el tamaño del folículo y un $35.2 \%$ mayor cuando aumenta $1 \mathrm{~mm}$ el tamaño de cuerpo lúteo.

\section{Discusión}

Las vacas Brown Swiss y sus cruzas con Bos indicus fueron las más adoptadas para la producción en la Amazonía ecuatoriana, aunque exista una gran necesidad de mejorar los indicadores reproductivos (Moyano y col., 2015). Dentro de un conjunto de problemas que poseen los rodeos, entre los más importantes es el retraso en el reinicio de la actividad ovárica después del parto (Guáqueta, Zambrano y Jiménez, 2014). Actualmente, hay disponible varios protocolos para aplicar en la Inseminación Artificial de Tiempo Fijo (IATF) para ser utilizados en vacas para carne y leche (Colazo y col., 2009; Bó, Baruselli y Mapletoft, 2018; Uslenghi y col., 2014), pero que necesitan ser revalidados en las condiciones de la Amazonía y en vacas Brown Swiss y sus cruzas con Bos indicus.

Los resultados de preñez obtenidos en este trabajo con J-Synch con y sin eCG, sobre 448 vacas Brown Swiss y sus cruzas inseminadas, 230 vacas se preñaron representando el $51.3 \%$, coincidiendo con los resultados reportados por Baruselli y col. (2005) y López (2001) en el trópico, también coinciden con encontrados por López y col. (2014) y Quinteros (2009) realizados en la Amazonía Ecuatoriana. Además, concuerdan los porcentajes de preñez con los obtenidos por otros investigadores utilizando similares protocolos de proestros prolongados J-Synch en la Amazonía, cuando se administró eCG y se inseminó a las 60 horas de retirado el dispositivo con el $61 \%(70 / 115)$ de preñez en comparación al inseminado a las 72 horas adicionando eCG, mostrando un 47\% (52/111) de preñez (López, 2017; YánezAvalos y col., 2018).

Los programas de inseminación artificial a celo detectado son afectados y poseen una baja eficiencia en el trópico, debido a que el celo posee una corta duración durante el día y una tendencia a expresarlo en la noche (López, 2014). La expresión de celo se mostró en un $51 \%$ del total de animales, por debajo del $90.4 \%$ reportado por López (2017) y De La Mata, Ré y Bó (2015). A su vez estos datos contrastan con los reportados por López (2017) quién mostró que el $78.2 \%$ de vacas Pardo Suizo mestizas presentaron celo al ser tratadas con el protocolo J-Synch en la Amazonía con un régimen alimenticio en pastoreo libre. Una de las explicaciones del bajo porcentaje de expresión del celo en las vacas en este tra- 
bajo podría ser que las mismas están atadas individualmente en espacios reducidos, no pueden expresar los síntomas previos del inicio del celo (grupo sexualmente activo), como es el roce y juego entre las vacas tratadas y no pueden montarse entre ellas, por consiguiente, la detección del celo se reduce a la observación subjetiva por parte del encargado de otros síntomas menos expresivos.

Tabla 6. Coeficientes estimados y método de máxima verosimilitud.

\begin{tabular}{cccccc}
\hline Parámetro & DF & Estimación & $\begin{array}{c}\text { Error } \\
\text { estándar }\end{array}$ & $\begin{array}{c}\chi^{2} \\
\text { de Wald }\end{array}$ & Pr $>\chi^{2}$ \\
\hline Intercept & 1 & -133.388 & 11.689 & 1.302 .273 & $<.0001$ \\
Protocolo & 1 & 14.499 & 0.2523 & 330.160 & $<.0001$ \\
Protocolo & 1 & 10.820 & 0.2369 & 208.638 & $<.0001$ \\
Protocolo & 1 & 12.468 & 0.2417 & 266.001 & $<.0001$ \\
Día & 1 & 13.733 & 0.2514 & 298.451 & $<.0001$ \\
TF mm & 1 & 0.4171 & 0.0614 & 461.369 & $<.0001$ \\
TCL mm & 1 & 0.3165 & 0.0368 & 739.256 & $<.0001$ \\
\hline
\end{tabular}

Los resultados de los diámetros de los folículos dominantes ovulatorios encontrados en este trabajo coinciden con los citados anteriormente por López (2017), en donde se mostró que el protocolo J-Synch tuvo el menor valor al retiro de $8.9 \pm 0.1 \mathrm{~mm}$, pero se evidenció mayor tamaño al momento de la IATF de $13.5 \pm 0.1 \mathrm{~mm}$, en comparación con el tratamiento Cipionato de Estradiol que presentó un mayor tamaño al retiro de $9.7 \pm 0.1 \mathrm{~mm}$ y $12.4 \pm 0.1 \mathrm{~mm}$ al momento de la IATF. El tratamiento Benzoato de Estradiol tuvo un tamaño de $9.5 \pm 0.1 \mathrm{~mm}$ al retiro del dispositivo y $12.9 \pm 0.1 \mathrm{~mm}$ a la IATF, siendo menores a lo alcanzando por el folículo dominante ovulatorio del tratamiento J-Synch.

Un experimento en Uruguay con vaquillonas para carne y en otro ambiente, mostraron que el grupo J-Synch tuvo un horario promedio de ovulación de $93.7 \pm 12.9$ horas (De La Mata, Ré y Bó, 2015). En este trabajo el horario promedio de ovulación para J-Synch con eCG a las 60 y 72 horas fue de $94.5 \pm 0.9$ horas y para J-Synch sin eCG a las 60 y 72 horas fue de $88.0 \pm 1.1$ horas. Esto coincide con los resultados encontrados por De La Mata, Ré y Bó (2015), quienes al utilizar un protocolo de J-Synch obtuvieron un promedio de ovulación de $93.7 \pm 12.9$ horas, y a los reportados por López (2017) quien al usar Brown Swiss en el mismo ambiente, obtuvo un intervalo de ovulación $87.7 \pm 0.6$ horas.

Los resultados del tamaño de los folículos dominantes ovulatorios encontrados en este trabajo mostraron que al retiro del dispositivo de progesterona existió diferencias significativas $(\mathrm{p} \leq 0.05)$ con mayor tamaño para $T 2(9.7 \pm 0.1 \mathrm{~mm})$; de la misma manera, el tamaño del folículo al momento de la inseminación artificial expresó diferencias significativas $(\mathrm{p} \leq 0.05)$ con mayor tamaño para T4 $(13.2 \pm$ $0.1 \mathrm{~mm}$ ) y al finalizar el estro el tamaño del folículo manifestó diferencias significativas $(\mathrm{p} \leq 0.05)$ con un tamaño mayor para T3 (13.9 $\pm 0.2 \mathrm{~mm})$. López (2017) reportó utilizando vacas Brown Swiss mestizas en la Amazonía y con un régimen alimenticio en pastoreo libre para el tratamiento J-Synch un tamaño al retiro de $8.9 \pm 0.1 \mathrm{~mm}$ y de $13.5 \pm 0.1 \mathrm{~mm}$ al momento de la inseminación artificial.

Yánez-Avalos y col. (2018), también utilizando vacas Brown Swiss en la Amazonía encontró que el tamaño del folículo fue para J-Synch con eCG 60 horas de $9.53 \pm 0.1 \mathrm{~mm}$ al momento de retirar el dispositivo de progesterona y de $9.87 \pm 0.1 \mathrm{~mm}$ para J-Synch con eCG $72 \mathrm{~h}$; mientras que, en el momento de la inseminación para J-Synch con eCG 60 horas fue de $12.3 \pm 0.1 \mathrm{~mm}$ y para J-Synch con eCG $72 \mathrm{~h}$ fue de $11.9 \pm 0.1 \mathrm{~mm}$, sin diferencias significativas. De La Mata, Ré y Bó (2015), encontró que la incorporación de eCG al protocolo J-Synch provoca una ventana mayor de tiempo para realizar la inseminación, y que, además, facilita la inseminación en grupos de animales mayores, en donde el grupo JSynch inseminadas a las 60 horas presentó porcentajes de preñez de $60.6 \%(\mathrm{P} \leq 0.05)$ comparadas con aquellas del mismo grupo que fueron inseminadas a las 72 horas. 
Tabla 7. Análisis de estimación de verosimilitud máxima

\begin{tabular}{cccc}
\hline Ratio de probabilidades & Estimación & \multicolumn{2}{c}{ Límites de confianza } \\
\hline Proto Con eCG 60 vs Con eCG 72 & 1.445 & 0.962 & 2.169 \\
Proto Con eCG 60 vs Sin eCG 60 & 1.225 & 0.805 & 1.865 \\
Proto Con eCG 60 vs Sin eCG 72 & 4.263 & 2.600 & 6.990 \\
Proto Con eCG 72 vs Sin eCG 60 & 0.848 & 0.562 & 1.279 \\
Proto Con eCG 72 vs Sin eCG 72 & 2.950 & 1.855 & 4.694 \\
Proto Sin eCG 60 vs Sin eCG 72 & 3.479 & 2.166 & 5.588 \\
día 6 vs 8 & 3.949 & 2.412 & 6.463 \\
TFmm & 1.518 & 1.345 & 1.712 \\
TCLmm & 1.372 & 1.277 & 1.475 \\
\hline
\end{tabular}

Núñez-Olivera y col. (2014) evaluaron el efecto sobre la prolongación del proestro y cómo éste incidía en el desarrollo del folículo ovulatorio cuando se les aplicaba una dosis de eCG y GnRH a las 48 y 72 horas luego de retirar el dispositivo, mostrando que a las 48 horas de retirado el dispositivo el diámetro fue de $8.8 \pm 0.4 \mathrm{~mm}$ y a la IATF de $12.3 \pm$ $0.5 \mathrm{~mm}$; a las 72 horas de retirado el dispositivo el diámetro fue de $8.2 \pm 0.3 \mathrm{~mm}$ y la IATF de $13.5 \pm$ $0.3 \mathrm{~mm}$ sin diferencia significativa.

Van-Eerdenburg y col. (2002) comprobó que las vacas que presentaban mayor tamaño de folículo, tenían una mayor probabilidad de ovular. En concordancia con lo anteriormente citado, Yánez y col. (2016) hallaron que vacas con un mayor tamaño folicular al momento de la inseminación artificial tuvieron catorce veces más posibilidades de quedar preñadas (preñadas: $13.4 \pm 1.1 \mathrm{~mm}$; vacías: $10.4 \pm$ $1.3 \mathrm{~mm})$.

En el trabajo se encontraron diferencias significativas $(\mathrm{p} \leq 0.05)$ en el tamaño del cuerpo lúteo al día 7 post inseminación entre los tratamientos utilizados. De La Mata, Ré y Bó (2015) encontró que el desarrollo luteal entre los días 4 y 13 posterior a la ovulación tendió a ser mayor $(\mathrm{P} \leq 0.074)$ en el grupo J-Synch comparado con el protocolo convencional. El tamaño del cuerpo lúteo estaría relacionado con la mayor o menor concentración sérica de progesterona que afectaría la fertilidad (Vasconcelos y col., 2001; Busch y col., 2008; Mann, 2009).

Trabajos anteriormente citados indicaron que la incorporación del protocolo J-Synch en condiciones ambientales extremas, permitió mejorar los índices de preñez en vacas con cría, anéstricas y con períodos abiertos extensos, en bovinos doble propósito y para carne en climas tropicales. Sin embargo, es necesario seguir sumando un número mayor de vacas Brown Swiss y sus cruzas, que es la vaca utilizada con mayor frecuencia en los sistemas producción en la Amazonía en los que se use el protocolo J-Synch, para consolidar los resultados.

\section{Conclusiones}

Los tratamientos J-Synch más eCG a las 60 horas y las 72 horas tuvieron diferente comportamiento en el desarrollo folicular y en el tamaño del cuerpo lúteo. El porcentaje de preñez no difirió entre los tratamientos. Aunque, existió un $44.5 \%$ más probabilidad de quedar preñada una vaca con el T1 en comparación con el T2. Además, aumentaría un 22.5\% la preñez aplicando el T1 en comparación con el T3, y sería un $18 \%$ mayor la preñez con el T3 si se compara con el T2.

\section{Referencias}

Baruselli, P. S. y col. (2005). «Introduçao da IATF no manejo reproductivo de rebanhos bovinos de corte no Brasil». En: $6^{\circ}$ Simposio Internacional de Reproducción Animal. Instituto de Reproducción Animal Córdoba. Córdoba, ARG., págs. 151-176.

Ben, G.A. y col. (2002). Manual de procedimientos. Programa de inseminación artificial a tiempo fijo. Manual. Sitio argentino de reproducción animal. Disponible en https:/ / bit.ly/39PI7Qd.

Bernardi, S.F. y col. (2011). «Evaluación de los cambios ocasionados en espermatozoides bovinos por variaciones en el manejo de las dosis durante su manipulación en inseminación artificial». En: InVet 13.2, 25-38. Online: https: / / bit.ly / 2GLMY8V. 
Bó, G. A., P. S. Baruselli y R. J. Mapletoft (2018). «Synchronization techniques to increase the utilization of artificial insemination in beef and dairy cattle». En: Animal Reproduction (AR) 10.3, 137-142. Online:https://bit.ly/3iWv6F2.

Bó, G.A. y col. (2018). «Programs for fixed-time artificial insemination in South American beef cattle.» En: Proceedings of the 10th International Ruminant Reproduction Symposium (IRRS 2018); Foz do Iguaçu, PR, Brazil.

Busch, D. C. y col. (2008). «Effect of ovulatory follicle size and expression of estrus on progesterone secretion in beef cows». En: Journal of animal science 86.3, 553-563. Online: https: / / bit.ly / 3iW7Wii.

Carrera Durazno, R., N. Fierro y J. Ordoñez (2015). Manual del pastoreo. Manual. Loja: Universidad Técnica particular de Loja.

Castellanos, S., I. Rentería y D. Maraña (2019). «Implementación del protocolo de inseminación artificial a tiempo fijo J-Synch en vacas brahman y F1 sin cría». En: Simposio Internacional de Reproducción Animal Pabellón Argentino. Córdoba, Argentina., 279. Online: https: / / bit.ly/3pTXigx.

Cedeño, A. y col. (2019). «Dinámica folicular y luteal en vaquillonas bos indicus de carne sincronizadas con el protocolo J-Synch de 7 días». En: Simposio Internacional de Reproducción Animal Pabellón Argentino. Córdoba, Argentina., 280. Online: https:/ / bit.ly/3pTXigx.

Colazo, M.G. y col. (2009). «Selección de los tratamientos hormonales disponibles en el mercado para la sincronización de celos en vaquillonas de carne». En: Resúmenes en CD del VIII Simposio Internacional de Reproducción Animal. Pabellón Argentino, Ciudad Universitaria, Córdoba.

De La Mata, J.J., M. Ré y G.A. Bó (2015). «Combination of estrus detection and fixed-time artificial insemination in beef heifers following shortened estradiol-based protocol that provides for a lengthened proestrus». En: Reproduction, fertility and development 27.1, 96-97. Online: https: / / bit.ly/3iWTgPZ.

De la Mata, J.J. y G.A. Bó (2012). «Sincronización de celos y ovulación utilizando protocolos de benzoato de estradiol y GnRH en períodos reducidos de inserción de un dispositivo con progesterona en vaquillonas para carne.» En: Taurus 55, 17-23. Online: https:/ / bit.ly/3nPEKgs.

González-Marcillo, R. y col., eds. (1997). Manual de pastos tropicales para la Amazonía ecuatoriana.
Programa de Ganadería Bovina y Pastos. Manual no. 33. Napo-Ecuador.: INIAP. Disponible en https:/ / bit.ly/36JbGB2.

Guáqueta, M., J. Zambrano y E. Jiménez (2014). «Risk factors for ovarian postpartum resumption in Holstein cows, under high tropical conditions». En: Revista MVZ Córdoba 19.1, 3970-3983. Online: https:/ / bit.ly/33VmsCu.

Kastelic, J.P., D.R. Bergfelt y O.J. Ginther (1990). «Relationship between ultrasonic assessment of the corpus luteum and plasma progesterone concentration in heifers». En: Theriogenology 33.6, 1269-1278. Online: https:/ / bit.ly/3iYhBoo.

López, J.C. (2014). «Evaluación de dos sales de estradiol sobre la tasa de ó a la IATF en vacas doble propósito en la Amazonía ecuatoriana».pp. 25. Tesis Especialización. Zipaquirá, Colombia: Instituto de Reproducción Animal de Córdoba, Argentina.

- (2017). «Comparación de protocolos de IATF convencionales con un protocolo con proestro pro-longado en vacas doble propósito en la Amazonía Ecuatoriana». pp 52. Online: https : / / bit.ly/2H2zYLI. Tesis de Maestría. Córdoba Argentina: Universidad de Córdoba.

López, J.C. y col. (2014). «Relación entre genotipos y preñez con un protocolo de inseminación artificial en vacas en la Amazonía Ecuatoriana». En: Revista Científica Biológico Agropecuaria Tuxpan 2.4, 885-890. Online: https: / bit.ly/3jZt7Bj.

López, M. V. (2001). «Evaluación de fecundidad en vacas Holstein Friesian inseminadas a diferentes tiempos del umbral detectado por el sistema heatime». pp 95. Tesis Grado. Sangolquí, Ecuador: Universidad Superior Politcnica del Ejército.

Mann, G.E. (2009). «Corpus luteum size and plasma progesterone concentration in cows». En: Animal reproduction science 115.1-4, 296-299. Online: https://bit.ly/3dnXqiC.

Moyano, J.C. y col. (2015). «Plasmaspiegel von LH (luteinisierendes Hormon), Brunstsymptome und Qualität der Gelbkörper in verschiedenen Protokollen, zur Synchronisation der Brunst in Brown-Swiss-Milchrindern». En: Züchtungskunde 4, págs. 265-271.

Núñez-Olivera, R. y col. (2014). «Ovulatory response and luteal function after eCG administration at the end of a progesterone and estradiol'based treatment in postpartum anestrous beef cattle». 
En: Animal reproduction science 146.3-4, 111-116. Online: https:/ / bit.ly/34U0NtY.

Quinteros, O.R. (2009). «La Inseminación Artificial

a Tiempo Fijo (IATF), con el protocolo Crestar (Norgestomet - Valerato de Estradiol)-Folligon (eCG)- Fertagil (Gonadorelin), como una alternativa reproductiva en la ganaderia del centro de la Amazonía Ecuatoriana». En: VII Seminario de Actualización Veterinaria de la Universidad de Ciencias Comerciales de Managua. Nicaragua, pág. 5.

Rodriguez, C., G. Saavedra y D. Gómez (2015). «Efecto de la etapa de lactancia sobre la calidad fisicoquímica de leche en vacas de raza Holstein y Normando». En: Zoot Trop 33, 23-35. Online: https://bit.ly/3iYYJWo.

Uslenghi, G. y col. (2014). «Effect of estradiol cypionate and amount of progesterone in the intravaginal device on synchronization of estrus, ovulation and on pregnancy rate in beef cows treated with FTAI based protocols». En: Animal reproduction science 145.1-2, 1-7. Online: https: / / bit.ly / 3dEAkEN.

Van-Eerdenburg, F.J. y col. (2002). «The relationship between estrous behavioral score and time of ovulation in dairy cattle». En: Journal of Dairy Science 85.5, 1150-1156. Online: https: / / bit.ly / 33Zeetp.

Vasconcelos, J. y col. (2001). "Reduction in size of the ovulatory follicle reduces subsequent luteal size and pregnancy rate». En: Theriogenology 56.2, 307-314. Online: https:/ / bit.ly/34YE2VF.

Yánez-Avalos, D. y col. (2018). «Inseminación artificial a tiempo fijo en vacas con proestro prolongado de 60 y 72 horas». En: Agronomía Mesoamericana 29.2, 363-373. Online: https: / / bit.ly / $31 \mathrm{aN} 2 \mathrm{pz}$

Yánez, D. y col. (2016). «Possible factors affecting pregnancy rate of cows in the amazon ecuatorian». En: Proceedings VI Peruvian Congress Animal Reproduction. SPERMOVA, pág. 66.

Yánez, D. y col. (2017). «Evaluación de un protocolo de IATF con proestro prolongado más eCG, sobre la tasa de preñez en vacas doble propósito de la Amazonía Ecuatoriana». En: Simposio Internacional de Reproducción Animal Pabellón Argentino. Córdoba, Argentina., 399. Online: https: //bit.ly/3pTXigx. 